\title{
Accuracy Assessment of HIPPARCOS Optical and VLA Radio Positions of Stars
}

\author{
H.G. Walter ${ }^{1}$, R. Hering ${ }^{1}$, H. Lenhardt ${ }^{1}$, Chr. deVegt ${ }^{2}$, \\ D.R. Florkowski ${ }^{3}$, K.J. Johnston ${ }^{4}$
}

\author{
${ }^{1}$ Astronomisches Rechen-Institut, Mönchhofstr. 12-14, \\ D-6900 Heidelberg, Germany \\ ${ }^{2}$ Hamburger Sternwarte, Gojenbergsweg 112, \\ D-2050 Hamburg 80, Germany \\ ${ }^{3}$ U.S. Naval Observatory, Washington, D.C. 20390, USA \\ ${ }^{4}$ Naval Research Laboratory, Washington, D.C. 20375, USA
}

\begin{abstract}
Optical positions of some 30 radio stars derived from 12 months of HIPPARCOS measurements are compared with their radio positions obtained with the Very Large Array (VLA). - Once the lengths of arcs between optical and radio positions of pairs of stars are calculated the differences of the arcs are formed. They provide an estimate of the coincidence of the optical and radio emission centres. - From the comparison of optical and radio positions infinitesimal rotation angles of the HIPPARCOS frame with respect to the VLA extragalactic reference frame are determined by rigid rotations. After taking account of the relative orientation of the frames the standard deviations of the remaining residuals are approximately of the order of the VLA observation errors, thus demonstrating the reliability of the HIPPARCOS results. However, they also indicate some data noise very likely caused by the low accuracy of optical proper motions used to bridge the HIPPARCOS-radio epoch differences up to 9 years, and possible discrepancies of radio-optical emission centres of some stars.
\end{abstract}

\section{Introduction}

For some 112000 stars 3-parameter solutions consisting of ecliptic longitude, ecliptic latitude and parallax have been obtained from 12 months of HIPPARCOS observations within the consortium FAST. Details on the procedures used are laid down in Kovalevsky et al. (1992). Among this set we identified 32 radio stars for which precise radio positions are available (Florkowski et al., 1985; deVegt and Johnston, 1992) that have been measured by means of the Very Large Array (VLA). On the hypothesis of coinciding optical and radio emission centres these radio positions, which have formal errors of about 30 milliarcseconds (mas), are potential candidates for assessing the astrometric performance of HIPPARCOS.

In a first step we estimate the coincidence of the emission centres by comparing the optical and radio length of arcs between pairs of stars. These arcs are expected to be nearly identical for an individual pair under the above hypothesis. - Because of possible systematic discrepancies 
of the HIPPARCOS frame and the VLA extragalactic frame of reference the angles of rigid rotations between both frames are determined from the position vectors of 32 stars. Finally, the discussion of the present state of accuracy centres on the residuals, radio minus optical, resulting from the application of the rotations to the HIPPARCOS positions.

\section{Comparison of optical and radio arcs}

To get some insight in the coincidence of the radio and optical emission centres we have calculated at Julian epoch 1992.0 the arc lengths for all possible pairs of stars using their radio and optical positions. Comparing the respective radio and optical arcs leads to a fair agreement. The mean difference of 496 arcs amounts to 3.9 mas. For the standard deviation of the difference of a radio arc and an optical arc, however, we get as much as 84 mas which is larger than expected and casts some light on possible deviations of the measured radiation centres and deteriorating effects caused by epoch transformations. As a matter of fact, up to 9 years had to be spanned on using proper motions having accuracies between \pm 5 and \pm 10 mas/yr.

\section{Comparison of optical and radio positions}

The comparison of HIPPARCOS and VLA positions is performed in equatorial coordinates at Julian epoch 1992.0, which is the reference epoch for astrometric results within the consortium FAST. To this end the HIPPARCOS positions have been transformed into equatorial coordinates while the VLA positions required an epoch transformation to 1992.0 which was executed by employing optimum optical proper motions at present available (Réquième, Mazurier, 1991; deVegt and Johnston, 1992).

Using all 32 radio stars the results summarized in Table 1 are obtained. The standard deviations contain the combined erros of the VLA positions at 1992.0 and the HIPPARCOS accuracies, the latter ones being below 5 mas on the average. Five stars out of this sample are visual double stars (e.g. Walter et al., 1990) having separations of a few arcsec and are as such troubling candidates for HIPPARCOS. On deleting these objects the statistics given in the left half of Table 1 remains essentially unaltered. Adopting an average epoch difference of 6 years and assuming $\pm 5 \mathrm{mas} / \mathrm{yr}$ or $\pm 10 \mathrm{mas} / \mathrm{yr}$ for the proper motions the accuracy of the VLA positions at 1992.0 will be \pm 42 and \pm 67 mas, respectively. This is in acceptable agreement with the results of Table 1. Furthermore, the diagrams of Fig. 1 do not display any noticeable systematic trends.

Table 1. Comparison of VLA and HIPPARCOS positions of 32 radio stars in the sense VLA-HIP at Julian epoch 1992.0; unit 1 mas.

\begin{tabular}{lcccc} 
& \multicolumn{2}{c}{ Before rotation } & \multicolumn{2}{c}{ After rotation } \\
& $\Delta \alpha \cos \delta$ & $\Delta \delta$ & $\Delta \alpha \cos \delta$ & $\Delta \delta$ \\
\hline $\begin{array}{l}\text { Unweighted } \\
\text { mean difference }\end{array}$ & -30 & -15 & 3 & -11 \\
$\begin{array}{l}\text { Standard } \\
\text { deviation }\end{array}$ & 60 & 62 & 56 & 60
\end{tabular}


It remains to be seen to which extent the comparison of positions is adulterated by differences of the extragalactic frame of the VLA observations and the HIPPARCOS frame. Confining the investigation to systematic differences in terms of rotation angles about the $\mathrm{x}_{-}, \mathrm{y}-$ and $\mathrm{z}$-axes we derive from the positions of 32 stars the angles rotating the HIPPARCOS frame into the extragalactic frame:

$$
\begin{aligned}
& \phi_{x}=-22.0 \pm 12.5 \mathrm{mas} \\
& \phi_{y}=-15.7 \pm 13.8 \mathrm{mas} \\
& \phi_{z}=-44.2 \pm 13.4 \mathrm{mas} .
\end{aligned}
$$

When applying this rotation to the HIPPARCOS positions the statistics improves insignificantly as shown in the right half of Table 1.
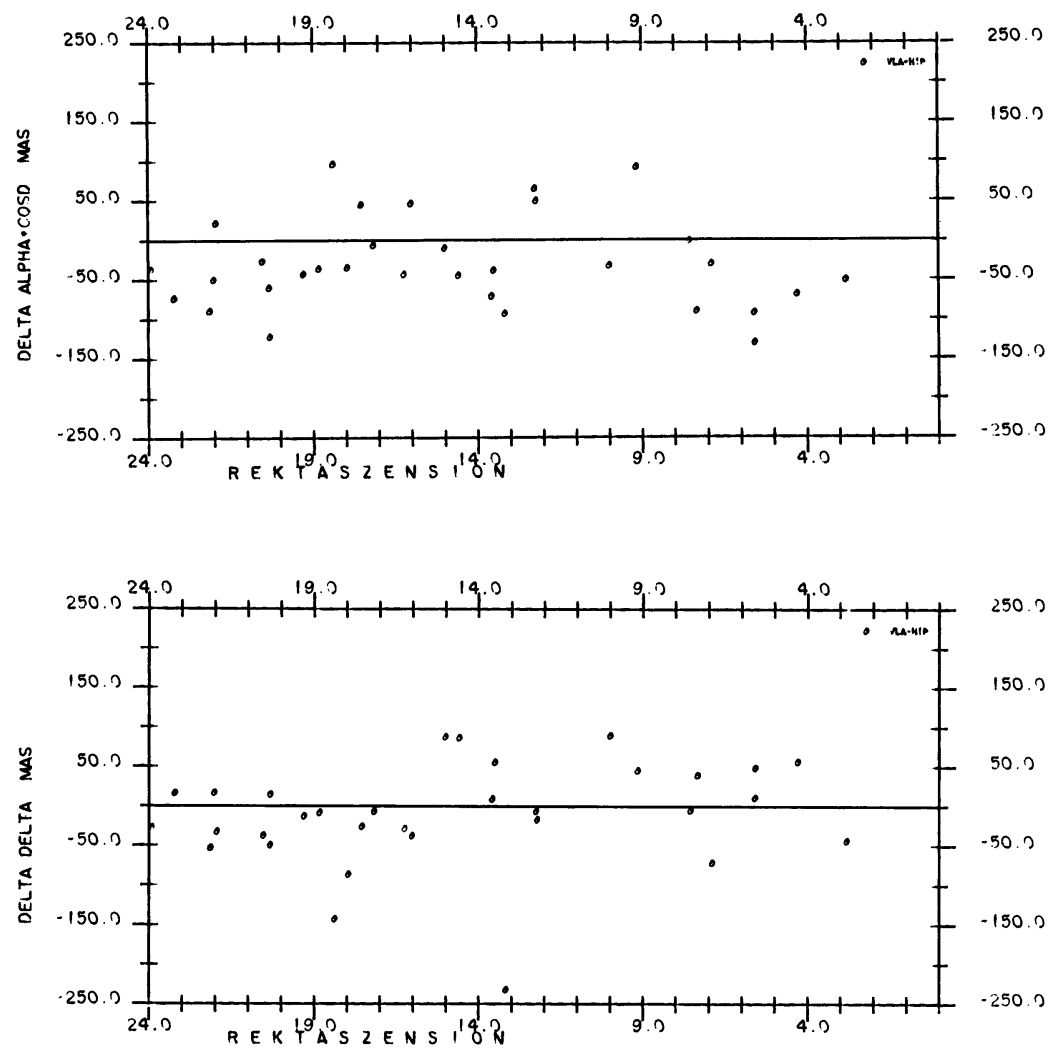

Fig. 1. Residuals VLA-HIP, before rotation into the extragalactic frame, at Jul. Epoch 1992.0.

On a star by star basis the residuals show surprisingly much scatter as illustrated in Fig. 1 where the residuals in right ascension and declination are plotted versus right ascension. 


\section{In Conclusion}

The comparison of VLA and HIPPARCOS positions yields standard deviations of the position differences which are mainly due to error propagation of catalogued ground-based proper motions to update the VLA positions to present epoch. In extreme cases 9 years of epoch difference had to be bridged. Nevertheless, it is inferred from the statistics of the radio stars that at least systematic gross errors of the optical data can be ruled out. From other tests is known that the HIPPARCOS position accuracy is at the level of a few mas. It would therefore make sense to ascribe the noise of the data to insufficient proper motions and to a possible non-coincidence of the radio and optical emission centres of particular stars to which the VLA and HIPPARCOS telescopes point. In this context the individual residuals of the radio and optical positions are informative. - The present comparison should be repeated as soon as accurate HIPPARCOSbased proper motions will become available. Then, the full quality of the VLA radio positions at their respective epochs can be maintained.

\section{Acknowledgements}

The financial support by the Bundesministerium für Forschung und Technologie (BMFT), Bonn, Germany, under the Project Nos. 500090020 and 50008810 is acknowledged.

\section{References}

deVegt, Chr. and Johnston, K.J., 1992, private communication

Florkowski, D.R., Johnston, K.J., Wade, C.W., deVegt, C., 1985, Astron. J. 90, 2381-2386

Kovalevsky, J., 1992, Astron. Astrophys. 258, 7-17

Réquième, Y., Mazurier, J.M., 1991, Astron. Astrophys. Suppl. Ser. 89, 311-318

Walter, H.G., Hering, R., deVegt, Chr., 1990, Astron. Astrophys. Suppl. Ser. 86, 357-393 